\title{
Mortalidad natural de Paracalanus indicus (Copepoda: Calanoida) en áreas de surgencia asociada a la zona de mínimo de oxígeno en el Sistema de Corrientes Humboldt: implicancias en el transporte pasivo del flujo de carbono
}

Natural mortality of Paracalanus indicus (Copepoda: Calanoida) in coastal upwelling areas associated with oxygen minimum zone in the Humboldt Current system: implications for the passive carbon flux

\section{Sonia Yáñez ${ }^{1,2}$, Pamela Hidalgo ${ }^{2,3}$ y Rubén Escribano ${ }^{2,3}$}

${ }^{1}$ Programa de Magister en Ecología de Sistemas Acuáticos, Facultad de Recursos del Mar, Universidad de Antofagasta, Angamos 601, Antofagasta, Chile

${ }^{2}$ Centro Oceanográfico del Pacifico Sur-Oriental (COPAS), Universidad de Concepción, Barrio Universitario s/n, casilla 160-C, Concepción, Chile

${ }^{3}$ Departamento de Oceanografía, Facultad de Ciencias Naturales y Oceanográficas, Universidad de Concepción, Barrio Universitario s/n, casilla 160-C, Concepción, Chile. sonyanez@udec.cl

\begin{abstract}
The in situ natural mortality (predation excluded) and growth rates $(g)$ of the pelagic copepod Paracalanus indicus were studied under variable upwelling regimes and upon presence of the oxygen minimum zone (OMZ) in two upwelling sites off Chile, Mejillones ( $\left.23^{\circ} \mathrm{S}\right)$ and Concepción $\left(\sim 36^{\circ} \mathrm{S}\right)$. Partial mortality was estimated using neutral red staining to distinguish live from dead copepods, and $g$ was estimated through the moulting rate method. Zooplankton was captured in austral summer and winter conditions during 2008-2010. Copepod abundance decreased in the OMZ, but the number and biomass of dead individuals increased in this layer. At Mejillones, $P$. indicus showed a population biomass of $160 \mathrm{mg}$ $\mathrm{C} \mathrm{m}^{-2}$, and $g$ between 0.1 and $0.4 \mathrm{~d}^{-1}$, (mean $\left.=0.24 \mathrm{~d}^{-1}\right)$. Production rates were about $35 \mathrm{mg} \mathrm{C} \mathrm{m}^{-2} \mathrm{~d}^{-1}$. Total flux of biomass from dead animals was $1.2 \mathrm{mg} \mathrm{C} \mathrm{m}^{-2} \mathrm{~d}^{-1}$, representing $3.4 \%$ of daily production. This contribution in organic C towards the $\mathrm{OMZ}$ may be nearly $0.67 \%$ of total passive flux of C. At Concepción, P. indicus produced a total biomass of $79 \mathrm{mg} \mathrm{C} \mathrm{m}^{-2}$. Growth rates $(g)$ were between $0.1-0.3 \mathrm{~d}^{-1}$, $\left(\mathrm{mean}^{-1}=0.23 \mathrm{~d}^{-1}\right)$ and $C$ production was $20 \mathrm{mg} \mathrm{C} \mathrm{m}^{-2} \mathrm{~d}^{-1}$, with a total biomass of dead animals of $0.2 \mathrm{mg} \mathrm{C} \mathrm{m}^{-2} \mathrm{~d}^{-1}$, representing $1.2 \%$ of daily production. This can represent about $0.13 \%$ of total passive $C$ sinking. Despite these rather low estimates, our study suggests that zooplankton as a whole should be considered as a significant source of freshly produced organic $C$ being incorporated and sustaining the $O M Z$ system in the upwelling zone.
\end{abstract}

Key words: Copepods, moulting rate, daily production, mortality

Resumen.- Se estudió la mortalidad natural (excluida la depredación) in situ y la tasa de crecimiento ( $g$ ) del copépodo epipelágico Paracalanus indicus en condiciones variables de surgencia, y en presencia de la zona de mínimo de oxígeno (ZMO) en dos centros de surgencia de Chile, Mejillones ( $\left.23^{\circ} \mathrm{S}\right)$ y Concepción $\left(\sim 36^{\circ} \mathrm{S}\right)$. La mortalidad parcial se estudió mediante tinción de rojo neutro para distinguir individuos vivos de aquellos muertos, y la tasa de crecimiento por estimaciones de tasas de muda. Se realizaron muestreos estratificados de zooplancton y observaciones oceanográficas en invierno y verano del 2009 al 2010. La abundancia total de copépodos disminuyó en la ZMO, mientras que la abundancia y biomasa de individuos muertos aumentó en esta capa. En Mejillones, P. indicus, mostró una biomasa poblacional de $160 \mathrm{mg} C$ $\mathrm{m}^{-2}$, y tasas de crecimiento $(\mathrm{g})$ entre 0,1 a $0,4 \mathrm{~d}^{-1}$, (promedio $=0,24 \mathrm{~d}^{-1}$ ). Las tasas de producción fueron cercanas a $35 \mathrm{mg} \mathrm{C} \mathrm{m}^{-2} \mathrm{~d}^{-1}$. La biomasa total de copépodos muertos fue de $1,2 \mathrm{mg} \mathrm{C} \mathrm{m}^{-2} \mathrm{~d}^{-1}$, representando un $3,4 \%$ de la producción diaria poblacional. Esta contribución de C orgánico hacia la ZMO puede representar un $0,67 \%$ del flujo vertical pasivo de C. En Concepción, P. indicus presentó una biomasa promedio de $79 \mathrm{mg} \mathrm{C} \mathrm{m}^{-2}$. Las tasas de crecimiento $(g)$ fueron entre $0,1-0,3 \mathrm{~d}^{-1}$, (promedio $=0,23 \mathrm{~d}^{-1}$ ) y la producción de $20 \mathrm{mg} \mathrm{C} \mathrm{m}^{-2} \mathrm{~d}^{-1}$, con una biomasa total de copépodos muertos de $0,2 \mathrm{mg} \mathrm{C} \mathrm{m}^{-2} \mathrm{~d}^{-1}$, representando un $1,2 \%$ de la producción diaria. Este aporte de C orgánico hacia la ZMO puede representar un $0,13 \%$ del flujo de $C$ pasivo. No obstante, los valores bajos obtenidos en este estudio sugieren que el zooplancton en su conjunto puede ser una fuente significativa de $\mathrm{C}$ orgánico recientemente producido que se incorpora y sustenta el funcionamiento del sistema de ZMO en el área de surgencia costera.

Palabras clave: Copépodos, tasas de muda, producción diaria, mortalidad 


\section{INTRODUCCIÓN}

El Sistema de Corrientes de Humboldt (SCH) se caracteriza por la presencia de una zona de mínimo oxígeno (ZMO), con bajos valores de oxígeno disuelto $\left(<0,5 \mathrm{ml} \mathrm{L}^{-1}\right)$. Esta ZMO se extiende desde el norte del Perú ( $\left.5^{\circ} \mathrm{S}\right)$ hasta el sur de Chile $\left(40^{\circ} \mathrm{S}\right)$ y sus variaciones en distribución, obedecen a cambios en regímenes de surgencia sobre el gradiente latitudinal. En el norte de Chile $\left(\sim 23^{\circ} \mathrm{S}\right)$, la surgencia es semi-permanente durante el año (Escribano 1998, Escribano et al. 2004), y la profundidad del borde superior de la ZMO, como promedio anual alcanza $26 \mathrm{~m}$ de profundidad (Hidalgo et al. 2005), mientras que en la zona centro/sur de Chile $\left(\sim 36^{\circ} \mathrm{S}\right)$ presenta un régimen marcadamente estacional con mayor ocurrencia durante el periodo de primavera-verano (Escribano et al. 2004, Sobarzo et al. 2007) y el borde superior de la ZMO se encuentra a la profundidad media anual de $44 \mathrm{~m}$ (Sobarzo et al. 2007).

La variabilidad de la surgencia y las variaciones en la profundidad de la ZMO en el SCH, pueden tener un efecto en la dinámica poblacional de las comunidades del zooplancton (Hidalgo et al. 2005, Hidalgo \& Escribano 2007, 2008) y en el transporte de bio-elementos entre las capas superficiales y la ZMO (Escribano et al. 2009). Dentro de la comunidad zooplanctónica de las zonas costeras del SCH, las especies de copépodos son el componente más abundante y representativo (Escribano \& Hidalgo 2000, Hidalgo \& Escribano 2001, Escribano et al. 2007, Hidalgo et al. 2010, 2012), manteniendo altas tasas de producción y altos niveles de producción secundaria (Escribano 1998, Escribano \& McLaren 1999, Hidalgo \& Escribano 2007, 2008). Paracalanus indicus es un copépodo calanoideo de pequeño tamaño ( $<1 \mathrm{~mm}$ longitud total) (Hidalgo et al. 2012), presenta altos niveles de abundancias y dominancia durante todo el año en las zonas de surgencia en las costas de Chile (Escribano \& Hidalgo 2000, Hidalgo \& Escribano 2001, Morales et al. 2010, Hidalgo et al. 2012), por lo cual puede representar un papel clave en la estructura y función de la comunidad zooplanctónica en las zonas de surgencias costeras del SCH. Estudios previos reportan que más del $46 \%$ de la abundancia total de los copépodos pelágicos del $\mathrm{SCH}$ corresponde a P. indicus (Hidalgo \& Escribano 2001, Escribano et al. 2001, Hidalgo et al. 2010, 2012), considerándose entonces, como especie modelo para estudios de dinámicas poblacionales y estimaciones de parámetros demográficos, tales como, estimaciones de tasas de mortalidad natural, que representen respuestas fisiológicas a la variabilidad ambiental del SCH (i.e., presencia y dinámica de la ZMO y variabilidad en los regímenes de surgencias costeras en el gradiente latitudinal). Sin embargo, estudios de abundancia de la población del zooplancton marino raramente consideran el estado vital de los organismos al momento del muestreo, mientras que los individuos muertos pueden comprender al menos un $10 \%$ del total de los individuos muestreados (Elliott \& Tang 2009). La presencia de individuos muertos representa un sustrato orgánico lábil para microorganismos (Tang et al. 2006), un potencial recurso alimentario para otros organismos (Zajaczkowskia \& Legezynska 2001), así como un vehículo para transportar biomoléculas esenciales y toxinas (Lee \& Fisher 1994, Frangoulis et al. 2005, Bickel \& Tang 2010). Una clara distinción entre organismos muertos y aquellos metabólicamente activos es necesaria para estimar la profundidad del hábitat de las especies (Escribano et al. 2009). Algunos estudios indican que los copépodos muertos pueden representar una gran fracción $(\sim 1 / 4-1 / 2)$ del flujo pasivo total de carbono orgánico particulado hacia aguas profundas (Sampei et al. 2009). Sin embargo, el aporte de materia orgánica hacia aguas profundas, derivada de la mortalidad natural de los organismos y su relación con la ZMO son inexistentes en zonas de surgencia costera en el SCH.

Las estimaciones de tasas de mortalidad natural, además de las limitaciones metodológicas existentes, tales como las tablas de vida, requieren largas series de tiempo y alta abundancia de los organismos (Aksnes et al. 1997, Ohman 2012). Además requieren de muchos supuestos, tales como que el reclutamiento diario de los estadios no debe tener tendencias muy bajas o muy altas, o que el transporte vertical es de muy baja influencia (Aksnes \& Ohman 1996), además de que la variabilidad ambiental pueda afectar estas estimaciones, principalmente en ambientes de surgencia. El método de tinción de rojo neutro, ajustado para las especies de copépodos (Tang et al. 2006, Elliott \& Tang 2009, Yañez 2009¹), puede permitir estimaciones de la mortalidad natural cuando se colectan los organismos. Sin embargo, esta metodología de estimación de mortalidad excluye el efecto de la

\footnotetext{
${ }^{1}$ Yáñez S. 2009. Tasa de mortalidad y desarrollo de Paracalanus cf. indicus (Copepoda: Calanoida) (Wolfender, 1905) en la zona Centro-Sur de Chile $\left(36^{\circ} \mathrm{S}\right)$ asociada con la zona de mínimo de oxígeno. Seminario de título, Facultad de Ciencias Naturales y Oceanográficas, Universidad de Concepción, Concepción, 65 pp.
} 
depredación sobre los organismos y considera aquellos factores que potencialmente pueden afectar los tamaños poblacionales de las especies, tales como, enfermedades bacterianas y virales, parasitismo, senescencia y toxicidad debido afloramientos fitoplanctónicos específicos (Poulet et al. 1995, 2007), o efectos de hipoxia o anoxia producto del ascenso de la ZMO (Escribano et al. 2009). El principio de la tinción de rojo neutro es teñir el aparato de Golgi (ARNm) de los organismos, el cual es determinante para identificar aquellos organismos metabólicamente activos, permitiendo discriminar entre los organismos vivos y muertos (Fleming \& Coughlan 1978).

El presente estudio utiliza el método de tinción de rojo neutro para determinar la distribución vertical de organismos vivos y muertos de $P$. indicus en relación a la ZMO en dos áreas de surgencias en las costas de Chile $\left(23^{\circ} \mathrm{S}\right.$ y $\left.36^{\circ} \mathrm{S}\right)$. Además de evaluar la contribución en $\mathrm{C}$ orgánico producto de la mortalidad natural de $P$. indicus hacia la profundidad de la ZMO, vía transporte pasivo. El estudio propone que la presencia de la ZMO constituye un factor que incrementa la mortalidad de esta especie, induciendo de esta manera un mayor flujo vertical de $\mathrm{C}$ hacia la ZMO vía transporte pasivo de organismos muertos.

\section{MATERIALES Y MÉTODOS}

\section{Trabajo en terreno}

El estudio se realizó en dos zonas de surgencia costeras, en el norte (Mejillones, $\sim 23^{\circ} \mathrm{S}$ ) y centro/sur de Chile (Concepción, $\sim 36^{\circ} \mathrm{S}$ ) del Pacífico Sur-Oriental en el SCH (Fig. 1). Se realizaron dos cruceros oceanográficos: En Mejillones, se realizó un crucero que muestreó 3 estaciones correspondientes a la serie de tiempo Mejillones, y en la zona centro/sur, se realizó un crucero, denominado BioZoo 06, que corresponde a un estudio estacional de la zona. La variabilidad espacial del muestreo de ambas zonas consistió en una transecta costa-océano con tres estaciones dentro del área de surgencia y en periodos de mayor intensificación (primavera-verano) de los eventos de surgencia, durante 2010-11 en el norte y 2009 zona centro/sur de Chile. En ambas zonas se muestrearon 3 estaciones, sin embargo, para efectos de este estudio sólo se analizaron 2. En el norte, fueron denominadas St2 y St3 de 75 y 90 m de profundidad y a 2 y 3 millas náuticas (mn) desde la costa, respectivamente. En la zona centro/sur se denominaron St. 5 y St.12 de 60 y $75 \mathrm{~m}$ de profundidad y a 5 y $12 \mathrm{mn}$ desde la costa, respectivamente. Estas estaciones reflejan las condiciones oceánicas de cada una de las zonas de estudios y evidencian la presencia de la zona de mínima de oxígeno.

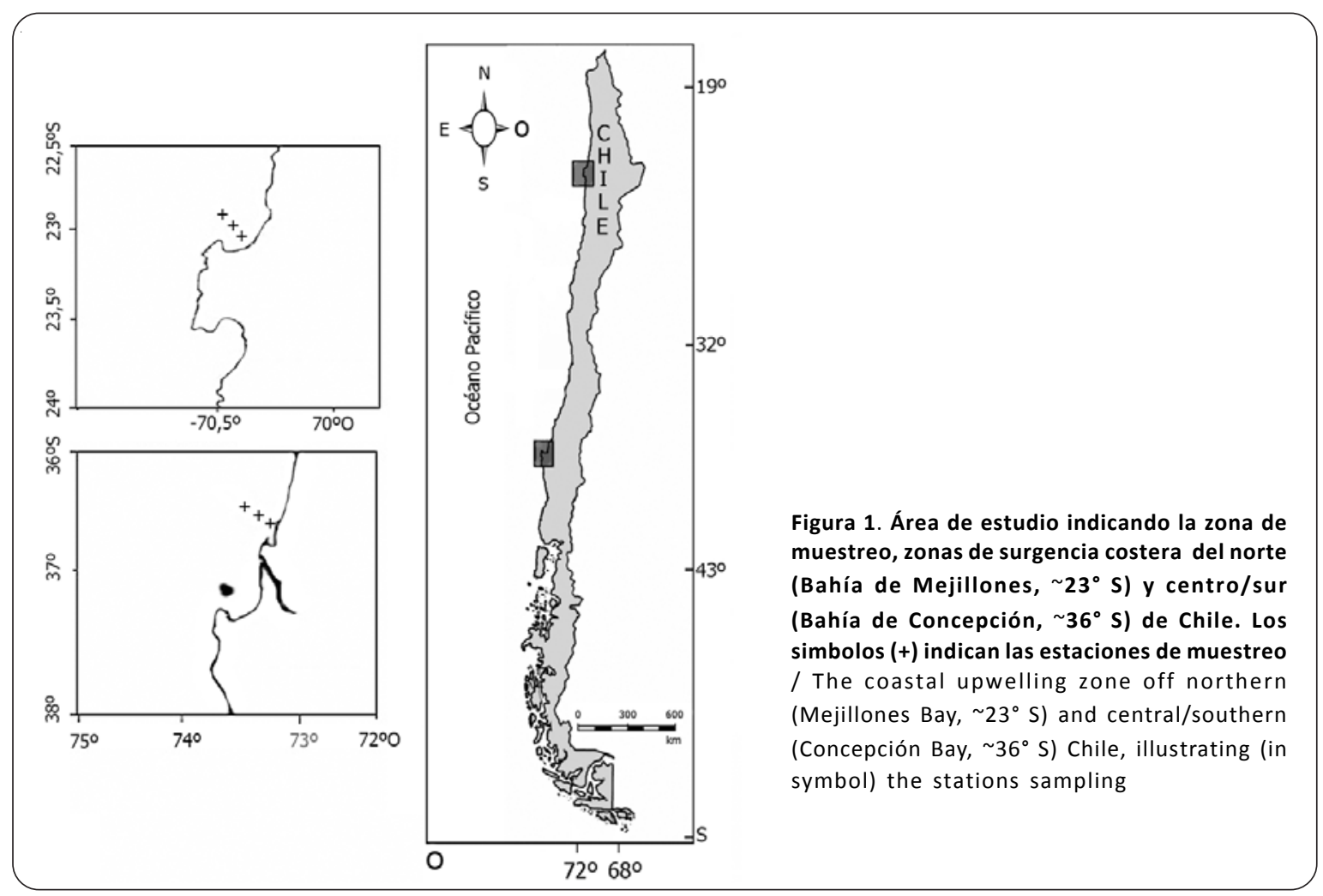

Vol. 47, №2, 2012 


\section{Hidrografía}

En los muestreos se obtuvo información de las condiciones ambientales de la columna de agua, tales como, temperatura $(\mathrm{T})\left({ }^{\circ} \mathrm{C}\right)$, salinidad (SAL) y contenido de oxígeno disuelto (OD) $\left(\mathrm{ml} \mathrm{L}^{-1}\right)$ en la columna de agua, mediante el uso de equipos oceanográficos autónomos, tipo CTD-O modelo SeaBird SBE-19. Adicionalmente, se recolectó muestra de agua de mar desde los $10 \mathrm{~m}$ de profundidad, para realizar estimaciones de la biomasa fitoplanctónica, expresada como Chla en $\mathrm{mg} \mathrm{m}^{-3}$, usando la técnica de fluorometría de acuerdo a Parsons et al. (1984). La información obtenida de los perfiles de oxígeno disuelto fue empleada para definir 3 estratos en la columna de agua: capa de mezcla, oxiclina y el borde superior de la zona de mínimo de oxigeno (ZMO), para asociarla con la distribución vertical de $P$. indicus. La capa mezcla, corresponde a la capa superior oxigenada de la columna de agua, la oxiclina corresponde a la capa del mayor gradiente vertical del contenido de oxígeno disuelto y el borde superior de la ZMO, corresponde a la profundidad en la cual se encuentran concentraciones de oxígeno disuelto de $1 \mathrm{ml} \mathrm{L}^{-1}$ (Escribano 1998, Hidalgo et al. 2005).

\section{Muestreo zooplanctónico para muestras PRESERVADAS}

Los muestreos zooplanctónicos consistieron en lances verticales estratificados de la columna de agua en cada una de las estaciones. En la zona norte, en Mejillones, se realizaron lances usando una red tipo WP-2 de $200 \mu \mathrm{m}$ de trama de malla y $50 \mathrm{~cm}$ de diámetro de apertura de boca de la red, mientras que en Concepción, se realizaron lances usando una red tipo Multinet de $200 \mu \mathrm{m}$ de trama de malla y $50 \mathrm{~cm}$ de ancho de apertura de boca de red. Ambas redes equipadas con un flujómetro digital para estimar el volumen filtrado por la red durante la captura de los organismos. Una vez recolectada la muestra, a bordo de la embarcación se procedió al tratamiento de las muestras de acuerdo a la técnica de tinción de rojo neutro ajustada para copépodos por Tang et al. (2006), Elliot \& Tang (2009) y específicamente para $P$. indicus por Yáñez (2009¹), para distinguir a los organismos vivos de los organismos muertos recolectados in situ. Las muestras fueron preservadas con solución de formalina neutralizada al 10\%, para su posterior análisis en laboratorio.

\section{MuESTREO ZOOPLANCTÓNICO PARA MUESTRAS VIVAS}

Los muestreos zooplanctónicos para muestras vivas fueron utilizados para las actividades experimentales de estimaciones de tasas de muda y de desarrollo en la zona norte (Mejillones) durante febrero-abril de 2010 y julioagosto de 2011 y en la zona centro/sur (Concepción) durante agosto-octubre de 2009, agosto-septiembre 2011 y enero de 2012 (Fig. 1). Se realizaron lances oblicuos de red tipo WP-2 de $110 \mu \mathrm{m}$ o $200 \mu \mathrm{m}$ de abertura de malla para recolectar todos los estadios de desarrollo de $P$. indicus. La muestra viva fue cuidadosamente colocada en cajas termo-reguladas para ser transportadas al laboratorio. Además, se recolectó agua de mar a 10 m de profundidad con botellas oceanográficas de 5 y 12 L tipo Niskin, para las incubaciones de los organismos.

\section{ANÁLISIS DE INFORMACIÓN}

Las muestras de zooplancton preservadas fueron analizadas de acuerdo a la técnica ajustada de la tinción de rojo neutro (Tang et al. 2006, Elliott \& Tang 2009, Yáñez 2009). Con un estereoscópico óptico (2x y 4x), se identificaron, cuantificaron y separaron todos los individuos (estadios de copepoditos y adultos) de $P$. indicus teñidos con la tinción de rojo neutro, como organismos vivos (color rojo) y aquellos no teñidos (incoloros) como organismos muertos.

\section{TASAs De DesarRollo}

Los experimentos de tasas de desarrollo en copépodos están basados principalmente en la presunción que los individuos recién capturados y mantenidos en incubaciones cortas (horas) manifiestan tasas de desarrollo y crecimiento similares a aquellas in situ donde han sido capturado (Runge \& Roff 2000). Se realizaron 22 experimentos durante agosto, septiembre y octubre de 2009 y enero de 2012 en la zona centro/sur en Concepción y 20 experimentos durante febrero, marzo y abril de 2010 y julio-agosto de 2011 en la zona Mejillones. Estos experimentos consistieron en seleccionar 10 organismos en buenas condiciones del mismo estadio en triplicado e incubarlos en agua de mar filtrada $(23 \mu \mathrm{m})$ en condiciones de temperatura controlada de $15^{\circ} \mathrm{C}$ durante $24 \mathrm{~h}$, de acuerdo a Runge \& Roff (2000). Los experimentos se realizaron en función de los estadios encontrados, en algunos se incubaron de todos los estadios, mientras que otras oportunidades se incubaron solo aquellos estadios encontrados, o bien aquellos que faltaban para completar la fase completa de los estadios para los experimentos. Posteriormente, al tiempo de incubación, se contabilizaron los organismos del estadio inicial, los organismos del estadio siguiente, y aquellos organismos que murieron durante la incubación, para cada uno de los experimentos realizados en ambas zonas de estudio. 
Las estimaciones de cambios en la frecuencia de estadios durante las incubaciones de cada uno de los experimentos, permitieron componer la función que describe la tasa de muda:

$$
T M=\left[\left(N_{i}-N_{j+1}\right) / N_{i}\right] / t
$$

donde, $T M$ es la tasa de muda $\left(\mathrm{d}^{-1}\right), t$ es el tiempo (horas), y $j+1$ son los estadios iniciales y subsecuentes, descontados los organismos muertos.

La tasa de desarrollo corresponde entonces, a la tasa de individuos (obtenida desde TM) que van ingresando en cada estadio de la población durante la escala diaria.

Las estimaciones de la tasa de crecimiento se derivaron de la ecuación según Runge \& Roff (2000):

$$
g=T M \times \operatorname{Ln}\left(P_{2} / P_{1}\right) \quad \text { (2), }
$$

donde $g$ es la tasa de crecimiento $\left(\mathrm{d}^{-1}\right), T M$ es la tasa de muda, $P_{2} y P_{1}$ son los pesos $(\mu \mathrm{g})$, del estadio final y estadio inicial, respectivamente. Estimaciones directas de los pesos de cada uno de los estadios, expresados como carbono (C) fueron obtenidos desde individuos colectados vivos, separados por estadios y secados a $60^{\circ} \mathrm{C}$ por $24 \mathrm{~h}$. La mediciones de C orgánico total se realizaron mediante un auto-analizador de CHN, de acuerdo a los estándares establecidos, los cuales fueron muy concordantes con los pesos obtenidos indirectamente de la regresión lineal definidas por Chisholm \& Roff (1990), para las especies de Paracalanidae.

Los datos de abundancia de todos los estadios, vivos y muertos, fueron estandarizados a individuos por metro cúbico (ind. $\mathrm{m}^{-3}$ ) para todos los estratos. Además, se estimaron en función a la proporción por estratos, las abundancias relativas de los organismos vivos y muertos. La tasa de mortalidad diaria fue estimada de acuerdo al valor de la pendiente de la curva encontrada desde la regresión aplicada entre la proporción de muertos por unidad de profundidad (capa de mezcla, oxiclina y ZMO). La tasa de mortalidad natural diaria fue definida como:

$$
m^{\prime}=P_{m} \times T_{d}
$$

donde $m$ ' es la tasa de mortalidad diaria, $P_{m}$ es la proporción de muertos por unidad de profundidad y $T_{d}$ es la tasa de decantación de un copépodo muerto, equivalente a $60 \mathrm{~m} \mathrm{~d}^{-1}$, estimada para Acartia tonsa (Kimmel et al. 2010). Esta ecuación (3) no considera pérdidas potenciales de individuos muerto que pudieran ser depredados, o que están expuestos a procesos de degradación. En este caso $m$ ' asume entonces que tales pérdidas no son importantes, o al menos que no afectan significativamente la estimación de $m$ '.

Las actividades experimentales permitieron calcular los componentes de las ecuaciones (1), (2) y (3), para las estimaciones de las tasas de desarrollo, de crecimiento y de mortalidad natural, respectivamente. La biomasa de la población ( $\mathrm{mg} \mathrm{C} \mathrm{m}^{-2}$ ) fue estimada a partir desde la abundancia numérica de cada uno de los estadios de la población de $P$. indicus encontrados en las muestras preservadas y multiplicado por el peso seco específico de cada uno de ellos. Los valores de peso específico fueron expresados como unidades de carbono correspondiente al $40 \%$ de su peso seco, de acuerdo a lo estimado para copépodos (Harris et al. 2000, Escribano et al. 2007). Los experimentos de tasas de desarrollo y crecimiento, permitieron estimar el valor de $g$ para todos los estadios, los cuales permitieron las estimaciones de la producción diaria $\left(\mathrm{mg} \mathrm{C} \mathrm{m}^{-2} \mathrm{~d}^{-1}\right)$ de la población del copépodo $P$. indicus, para ambas zonas de estudio. El flujo vertical pasivo de carbono de $P$. indicus hacia la ZMO, se estimó a partir de la proporción de su biomasa total decantada (biomasa de los organismos muertos) por día y la proporción de esta biomasa con el valor de biomasa total transportada de la comunidad zooplanctónica, estimada por González et al. (1998), cuyo valor fluctúa entre 0,125 y $0,176 \mathrm{~g} \mathrm{C} \mathrm{m}^{-2} \mathrm{~d}^{-1}$.

Se realizaron análisis de varianza (ANOVA) de una vía para determinar diferencias de las condiciones oceanográficas entre las zonas de estudio y entre las 3 capas preestablecidas (capa de mezcla, oxiclina y ZMO) de la columna de agua de la zona de Mejillones y Concepción.

Para evidenciar asociaciones entre la distribución vertical de la abundancia de $P$. indicus, la profundidad de la ZMO y las estimaciones de flujo vertical de C (vía abundancia de organismos muertos), se realizó un test no-paramétrico Kruskal-Wallis. Para verificar diferencias entre las estimaciones de $g$ para cada estadio de desarrollo, entre estratos y entre zonas de estudios, se aplicaron ANOVA de una vía. Posteriormente, un análisis multivariado (MANOVA) fue utilizado para evaluar la influencia de la distribución de los estadios por estratos, sobre las abundancias de organismos vivos y muertos, y sobre las estimaciones de tasa de mortalidad para cada una las zonas de estudio. 


\section{Resultados}

\section{VARIABILIDAD OCEANOGRÁFICA}

Los resultados obtenidos de las condiciones oceanográficas ( $\mathrm{T}^{\circ}$, S y OD) de las estaciones muestreadas en cada una de las zonas de estudios evidenciaron la presencia de ZMO muy somera $(<50 \mathrm{~m})$ y una estratificación térmica y salina definida, tanto para la zona norte (Mejillones, $\sim 23^{\circ} \mathrm{S}$ ), como la zona centro/sur (Concepción, $\sim 36^{\circ} \mathrm{S}$ ) de Chile (Fig. 2). En la zona norte, la distribución de la temperatura en la columna de agua, mostró una capa de mezcla por sobre los $17 \mathrm{~m}$ de profundidad con valores registrados entre los $13^{\circ} \mathrm{C}$ y $17^{\circ} \mathrm{C}$, mientras que en la zona de Concepción se observó una columna de agua más homogénea, pero con una capa de mezcla por sobre los $19 \mathrm{~m}$ de profundidad con valores registrados entre $11^{\circ} \mathrm{C}$ y $12^{\circ} \mathrm{C}$. En ambas zonas, por debajo de la capa de mezcla, la temperatura se distribuyó homogéneamente entre la oxiclina y la ZMO (Fig. 2a, Tabla 1). La salinidad mostró un patrón estable de distribución vertical en la columna de agua en la zona norte, mientras que en Concepción, se registraron valores bajos en la capa superficial por sobre los $3 \mathrm{~m}$ de profundidad, homogeneizándose hacia las capas profundas (Fig. 2b, Tabla 1). El contenido de oxígeno disuelto en ambas zonas, mostró un patrón similar, con una capa superficial oxigenada por sobre los $20 \mathrm{~m}$ de profundidad, disminuyendo hasta valores registrados inferiores a $1 \mathrm{ml}$ $\mathrm{L}^{-1}$ hacia la capa profunda (Fig. 2c, Tabla 1).

La información obtenida de las variables oceanográficas se resume en la Tabla 1; los valores registrados de las condiciones ambientales permitió caracterizar la columna de agua en 3 estratos (capa de mezcla, oxiclina y ZMO), con la finalidad de estimar la distribución y abundancia de $P$. indicus en cada uno de ellos. El ANOVA mostró que existieron diferencias significativas entre los estratos de la columna de agua, definidos como capa de mezcla, oxiclina y ZMO, mientras que entre las zonas de estudios no se evidenciaron diferencias significativas entre los estratos correspondientes ni entre las zonas de estudio (Tabla 1). Por lo cual, para evaluar los efectos entre las proporciones de la distribución y abundancia de los organismos vivos y muertos de $P$. indicus y las variables ambientales

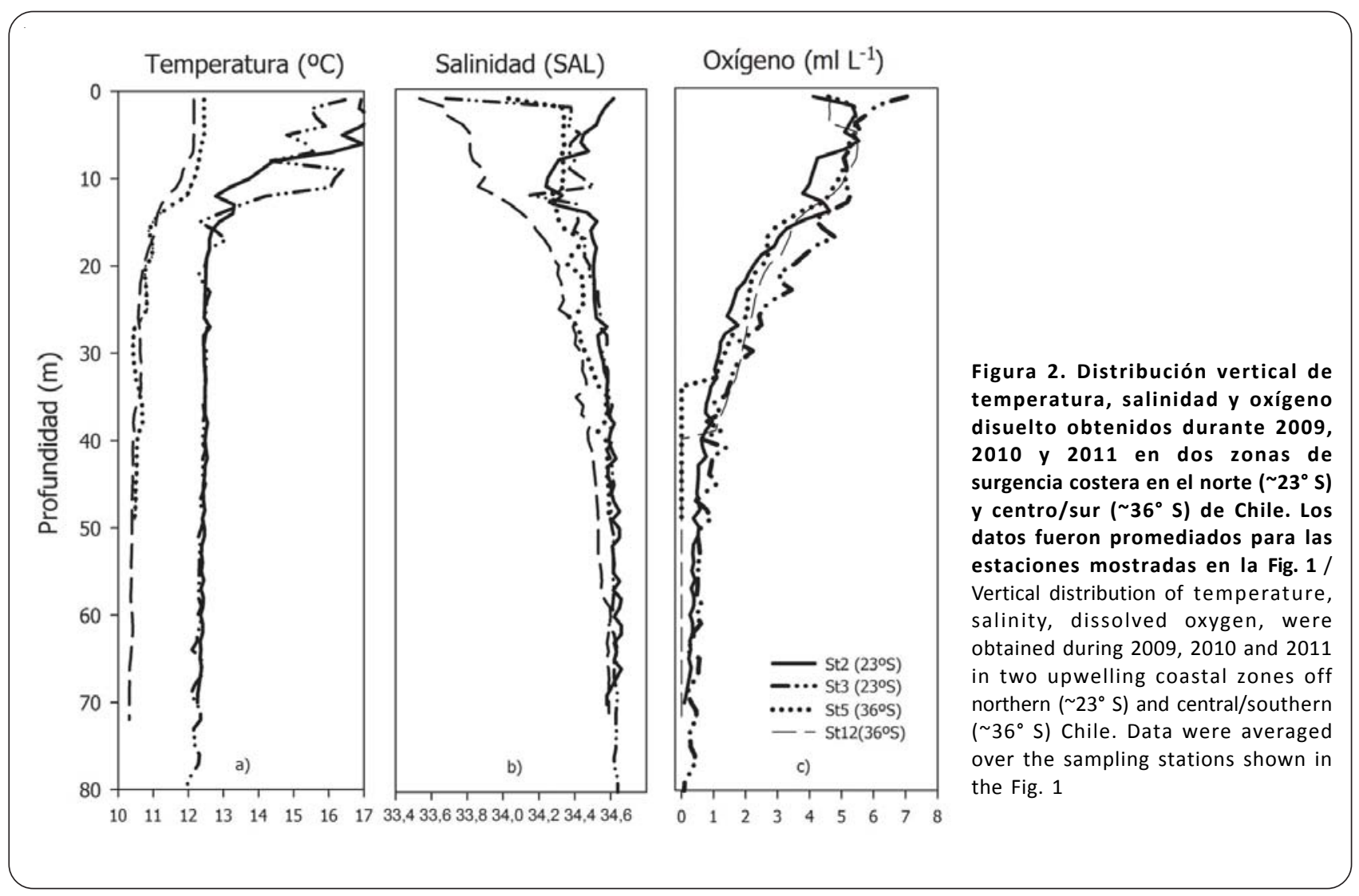


Tabla 1. Profundidad de la capa de mezcla, oxiclina y del límite superior de la zona de mínimo de oxígeno (ZMO), valores promedio y desviación estándar ( \pm ) de las variables oceanográficas, temperatura $(\mathrm{T})\left({ }^{\circ} \mathrm{C}\right)$, salinidad (SAL), oxígeno disuelto (DO) $\left(\mathrm{ml} \mathrm{L}^{-1}\right)$ y Clorofila (Chla) $\left(\mathrm{mg} \mathrm{m}^{-3}\right)$ de las estaciones muestreadas en la zona norte (Bahía de Mejillones, $\sim 23^{\circ} \mathrm{S}$ ) y centro/sur de Chile (Bahía de Concepción, $\sim \mathbf{3 6}^{\circ} \mathrm{S}$ ) / Depth of the mixed layer, oxycline and upper boundary of the $\mathrm{OMZ}$, mean and standard deviation $( \pm)$ of the oceanographic variables, temperature $(\mathrm{T})\left({ }^{\circ} \mathrm{C}\right)$, salinity (SAL), dissolved oxygen (DO) ( $\left.\mathrm{ml} \mathrm{L}^{-1}\right)$ and Chlorophyll (Chla) $\left(\mathrm{mg} \mathrm{m}^{-3}\right)$ from stations sampled in zone off northern (Mejillones Bay, $\sim 23^{\circ} \mathrm{S}$ ) and central-southern Chile (Concepción Bay, $\sim 36^{\circ} \mathrm{S}$ )

\begin{tabular}{|c|c|c|c|c|c|c|}
\hline & Variable & Capa de mezcla & Oxiclina & & MO & \\
\hline \multicolumn{7}{|c|}{ Bahía de Mejillones $\left(\sim 23^{\circ} \mathrm{S}\right)$} \\
\hline \multirow[t]{5}{*}{ St2 } & Z & $0-15$ & $15-32$ & \multicolumn{3}{|c|}{32} \\
\hline & $\mathrm{T}$ & $15,07 \pm 1,83$ & $12,51 \pm 0,07$ & 12,42 & \pm & 0,07 \\
\hline & SAL & $34,43 \pm 0,13$ & $34,52 \pm 0,03$ & 34,62 & \pm & 0,03 \\
\hline & DO & $4,63 \pm 0,64$ & $1,89 \pm 0,70$ & 0,50 & \pm & 0,24 \\
\hline & Chla & $15,28 \pm 13,14$ & $4,66 \pm 10,54$ & 0,89 & \pm & 0,22 \\
\hline \multirow[t]{5}{*}{ St3 } & $\mathrm{Z}$ & $0-17$ & $17-36$ & \multicolumn{3}{|c|}{36} \\
\hline & $\mathrm{T}$ & $14,88 \pm 1,35$ & $12,49 \pm 0,07$ & 12,31 & \pm & 0,13 \\
\hline & SAL & $34,35 \pm 0,19$ & $34,54 \pm 0,05$ & 34,62 & \pm & 0,01 \\
\hline & DO & $5,31 \pm 0,66$ & $2,45 \pm 0,91$ & 0,57 & \pm & 0,31 \\
\hline & Chla & $13,10 \pm 6,51$ & $2,51 \pm 1,98$ & 0,71 & \pm & 0,14 \\
\hline Promedios & $\mathrm{T}$ & $16,10 \pm 0,60$ & $12,50 \pm 0,04$ & 12,30 & \pm & 0,08 \\
\hline $\mathrm{St} 2+\mathrm{St} 3$ & DO & $5,40 \pm 0,20$ & $2,20 \pm 0,25$ & 0,50 & \pm & 0,11 \\
\hline \multicolumn{7}{|c|}{ Bahía de Concepción $\left(\sim 36^{\circ} \mathrm{S}\right)$} \\
\hline \multirow[t]{5}{*}{ St5 } & $\mathrm{Z}$ & $0-16$ & $16-33$ & \multicolumn{3}{|c|}{33} \\
\hline & $\mathrm{T}$ & $12,02 \pm 0,55$ & $10,68 \pm 0,21$ & 10,54 & \pm & 0,07 \\
\hline & SAL & $34,31 \pm 0,08$ & $34,44 \pm 0,04$ & 34,58 & \pm & 0,02 \\
\hline & DO & $4,74 \pm 0,78$ & $1,95 \pm 0,55$ & 0,05 & \pm & 0,00 \\
\hline & Chla & $2,76 \pm *$ & $*$ & & $*$ & \\
\hline \multirow[t]{5}{*}{ St12 } & Z & $0-19$ & $19-39$ & \multicolumn{3}{|c|}{39} \\
\hline & $\mathrm{T}$ & $11,63 \pm 0,52$ & $10,60 \pm 0,08$ & 10,38 & \pm & 0,04 \\
\hline & SAL & $33,94 \pm 0,22$ & $34,40 \pm 0,06$ & 34,58 & \pm & 0,03 \\
\hline & DO & $4,51 \pm 0,83$ & $1,91 \pm 0,49$ & 0,05 & \pm & 0,31 \\
\hline & Chla & $1,19 \pm *$ & $*$ & & $*$ & \\
\hline Promedios & $\mathrm{T}$ & $11,80 \pm 0,30$ & $10,60 \pm 0,05$ & 10,46 & \pm & 0,11 \\
\hline $\mathrm{St} 2+\mathrm{St} 3$ & SAL & $4,60 \pm 0,20$ & $1,93 \pm 0,03$ & 0,50 & \pm & 0,03 \\
\hline
\end{tabular}

* corresponden a valores no determinados

(temperatura y oxígeno disuelto), se definieron los valores promedios para determinar cada uno de los 3 estratos preestablecidos en ambas zonas. En la zona norte de Chile $\left(\sim 23^{\circ} \mathrm{S}\right)$ las temperaturas promedios en la capa de mezcla fue de $15,0^{\circ} \mathrm{C}, 12,5^{\circ} \mathrm{C}$ en la oxiclina y $12,4^{\circ} \mathrm{C}$ en la $\mathrm{ZMO}$, mientras que la zona centro/sur de Chile $\left(\sim 36^{\circ} \mathrm{S}\right)$ las temperaturas promedios de la capa de mezcla fue de $11,81^{\circ} \mathrm{C}, 10,64^{\circ} \mathrm{C}$ en la oxiclina y $10,5^{\circ} \mathrm{C}$ en la ZMO. Los valores promedios de la concentración de oxígeno disuelto en la capa de mezcla, oxiclina y ZMO, en la zona norte fueron de 5,0; 1,2 y $0,5 \mathrm{ml} \mathrm{O}_{2} \mathrm{ml} \mathrm{L}^{-1}$, y en zona centro/sur de Chile, de 4,62; 1,93 y $0,5 \mathrm{ml} \mathrm{O}_{2} \mathrm{ml} \mathrm{L}^{-1}$, respectivamente (Tabla 2).

\section{Distribución Vertical de Paracalanus indicus}

Se analizó la distribución vertical de los organismos vivos y muertos del copépodo $P$. indicus en relación a los tres estratos definidos por las condiciones oceanográficas durante los periodos de muestreos como capa de mezcla, oxiclina y ZMO (Tabla 1), para las estaciones muestreadas en ambas zonas (Tabla 2). La distribución vertical de $P$. indicus, mostró las mayores abundancias de los organismos vivos en la capa de mezcla (> 250 ind. $\left.\mathrm{m}^{-3}\right)$ y en la ZMO ( $>43$ ind. $\mathrm{m}^{-3}$ ), mientras que en la oxiclina la abundancia de los organismos vivos registró los menores valores ( 30-40 ind. $\mathrm{m}^{-3}$ ), a excepción de la estación St. 12 
Tabla 2. Valores totales integrados en la columna de agua de abundancia numérica (ind. $\mathbf{m}^{-3}$ ) de copépodos vivos y muertos, y abundancia de copépodos muertos sobre abundancia total (M/T) para los tres estratos muestreados en las estaciones de la zona norte (Bahía de Mejillones, $\sim 23^{\circ}$ S) y centro/sur de Chile (Bahía de Concepción, $\mathbf{3 6}^{\circ}$ S) / Total integrated live and dead copepod abundances (ind. $\mathrm{m}^{-3}$ ), and percent dead copepod (M/T) from three layer were sampled in the stations sampling at zone off northern (Mejillones Bay, $\sim 23^{\circ} \mathrm{S}$ ) and centralsouthern Chile (Concepción Bay, $36^{\circ} \mathrm{S}$ )

\begin{tabular}{ccccc}
\hline & & Capa de mezcla & Oxiclina & ZMO \\
\hline Bahía de Mejillones $\left(\sim 23^{\circ} \mathrm{S}\right)$ & & & & \\
St2 & Vivos & 313,4 & 53,3 & 309,3 \\
& Muertos & 1,2 & 7,2 & 34,3 \\
& M/T & 0,004 & 0,12 & 0,10 \\
St3 & & & & \\
& Vivos & 1058,9 & 30,7 & 6,1 \\
& Muertos & 28,4 & 1,5 & 9,1 \\
Bahía de Concepción $\left(\sim 36^{\circ} \mathrm{S}\right)$ & M/T & 0,03 & 0,05 & 0,6 \\
St5 & & & & \\
& Vivos & 409,23 & 43,3 & 43,9 \\
& Muertos & 31,7 & 31,2 & 28,3 \\
& M/T & 0,07 & 0,4 & 0,4 \\
St12 & & & & \\
& Vivos & 253,2 & 295,8 & 106,5 \\
& Muertos & 6,8 & 8,03 & 21,8 \\
& M/T & 0,03 & 0,03 & 0,2 \\
\hline
\end{tabular}

en Concepción, que se registraron altos valores en la capa intermedia. La distribución vertical de los organismos muertos mostró incrementos en su abundancia en la oxiclina y ZMO en las estaciones St. 2 y St. 12, mientras que las estaciones St. 3 y St. 5, mostraron un patrón de distribución opuesto. Sin embargo, cuando se examina la relación de los organismos vivos y el total de los organismos muestreados de la población (abundancia total) de $P$. indicus en cada uno de los estratos, se observó que la razón de organismos muertos y el total se incrementa hacia las capas profundas (oxiclina y ZMO) en todas las estación estudiadas (Tabla 2). La Figura 3 representa la distribución vertical de los organismos vivos y muertos y la razón de organismos muertos sobre la abundancia total de la población muestreada de $P$. indicus. En la zona norte de Mejillones, la estación St. 2, mostró la mayor abundancia de organismos vivos en la capa de mezcla, disminuyendo notoriamente hacia la oxiclina y ZMO (Fig. 3a), a diferencia de la estación St. 3 donde la mayores abundancias estuvieron en la capa de mezcla, disminuyendo en la ZMO (Fig. 3b). Cuando se analiza la distribución de las proporciones entre individuos vivos y muertos, se observó para ambas estaciones la presencia de organismos vivos y muertos en los tres estratos, con incrementos de organismos muertos hacia la oxiclina y ZMO (Fig. 3a y 3b). En la zona de Concepción, la St. 5, las mayores abundancias de los organismo vivos se observó en la capa de mezcla y ZMO (Fig 3c) mientras que en St. 12, la mayor abundancia se observó en la capa de mezcla, disminuyendo notablemente hacia las capas profundas (Fig. 3d). La relación entre organismos vivos y muertos con respecto a la abundancia total indicó que la proporción de individuos muertos en la capa de mezcla fueron bajos $(<10 \%)$, mientras que la oxiclina y ZMO, la proporción de muertos se incrementó notoriamente entre $20 \%$ y $30 \%$ para la zona de Mejillones y entre 10 y 60\% para la zona de Concepción (Fig. 3e-h).

\section{DISTRIBUCIÓN VERTICAL Y ABUNDANCIA DE COPEPODITOS Y ADULTOS DE PARACALANUS INDICUS}

La distribución general de los organismos vivos y muertos mostró un patrón similar en todos los estadios, con aumento de la abundancia de individuos muertos hacia las capas más profundas, tanto en la zona norte $\left(\sim 23^{\circ} \mathrm{S}\right)$ como centro/sur ( $\left.36^{\circ} \mathrm{S}\right)$ de Chile (Fig. 4). En la Tabla 3 se resume las abundancias numéricas y relativas de cada uno de los estadios de copepoditos y adultos de $P$. indicus, encontrados en los 3 estratos muestreados. 
Figura 3. Distribución vertical y abundancia total de individuos vivos y muertos de Paracalanus indicus con respecto a los estratos definidos en la St2 y St3 en el norte ( 23ㅇ)(a y b) y en la St5 y St12, centro/sur de Chile ( 36-S) (c y d); Abundancia relativa (\%) de copépodos muertos con respecto a la abundancia total en el norte ( 23oS) (e y f) y centro/ sur de Chile ( 369S) (g y h) / Vertical distribution and total live/dead copepods abundance of Paracalanus indicus with respect to three layer were sampled in the St2 and St3 at northern ( 23ㅇ) (a and b) and in the St5 y St12, centralsouthern Chile ( 36ㅇ) (c and d); Relative abundance (\%) of dead copepods on total abundance at northern ( 23ㅇ) (e and f) and central/southern Chile ( 36ㅇ) (g and $h$ )

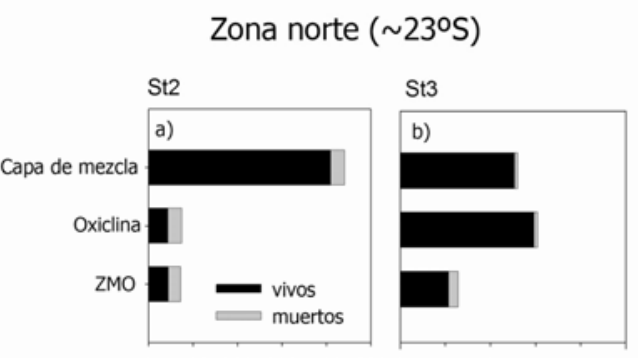

$0 \quad 100 \quad 200300400 \quad 500 \quad 0 \quad 100 \quad 200 \quad 300 \quad 400 \quad 500$

Abundancia (ind $\mathrm{m}^{-3}$ )
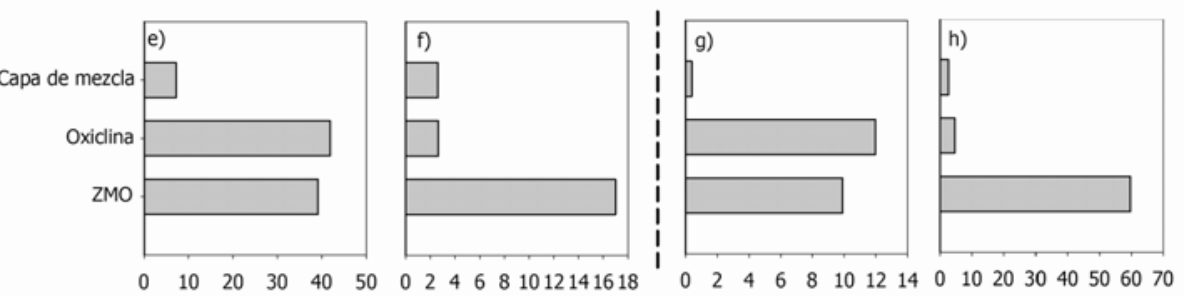

Abundancia relativa (\%)
Figura 4. Distribución vertical y abundancia relativa (\%) de copepoditos y adultos de Paracalanus indicus. Los estadios C1, C2, C3, C4, C5 y adultos en relación a los estratos definidos: capa de mezcla, a); oxiclina, b); ZMO, c) para el norte $\left(\sim 23^{\circ} \mathrm{S}\right)$ y capa de mezcla, d); oxiclina, e); ZMO, f) para la zona centro/sur de Chile ( $\left.36^{\circ} \mathrm{S}\right)$. La línea continua corresponde al perfil de oxígeno disuelto encontrado para ambas zonas de surgencia / Copepodid stages and adults vertical distribution and relative abundance (\%) of Paracalanus indicus. The copepodid stages $(\mathrm{C} 1, \mathrm{C} 2, \mathrm{C} 3, \mathrm{C} 4, \mathrm{C} 5)$ and adults with respect to the layers in the column waters. Oxygenated upper layer, a); oxycline, b); OMZ, c) at zone off northern ( $23^{\circ}$ S) and oxygenated upper layer, d); oxycline, e); ZMO, f) at zone off central/southern Chile $\left(\sim 36^{\circ} \mathrm{S}\right)$. Oxygen profile is illustrating (continuous line) for both upwelling coastal zones

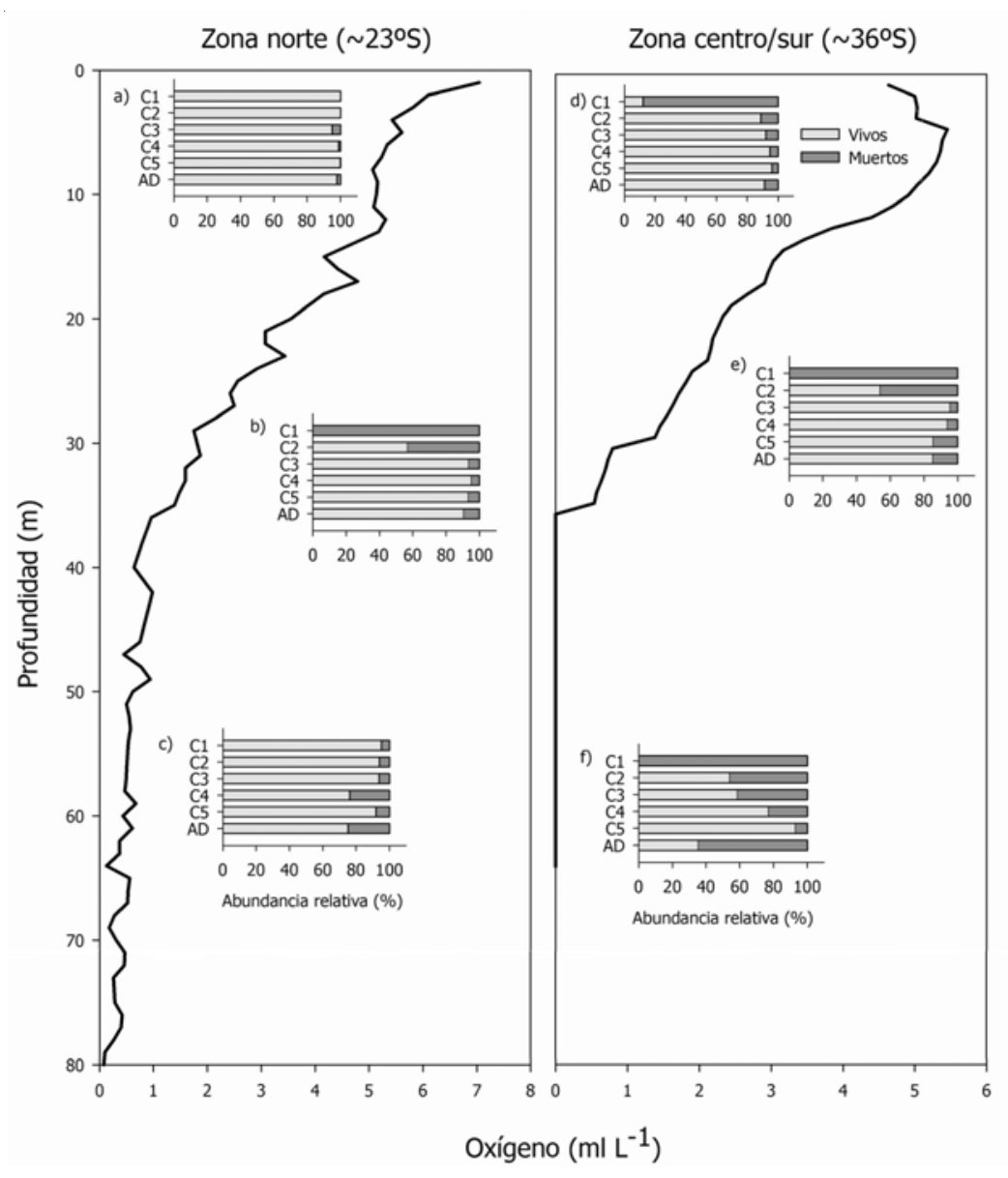

Vol. 47, №2, 2012 
Tabla 3. Tasas de desarrollo $\left[\mathrm{TM}\left(\mathrm{d}^{-1}\right)\right]$, crecimiento $\left[g\left(\mathrm{~d}^{-1}\right)\right]$, sus valores promedio y desviación estándar ( \pm ), para cada experimento, por estadio obtenidas mediante el método de tasas de muda en la zona norte $\left(\sim 23^{\circ} \mathrm{S}\right)$ y centro/sur de Chile ( $\left.\sim 36^{\circ} \mathrm{S}\right) /$ Development $\left[T M\left(d^{-1}\right)\right]$ and growth rates $\left[g\left(d^{-1}\right)\right]$, their mean and standard deviation $( \pm)$, from each experiments, for copepodid stages estimated through the molting rates method in the zone off northern $\left(\sim 23^{\circ} \mathrm{S}\right)$ and central-southern Chile $\left(\sim 36^{\circ} \mathrm{S}\right)$

\begin{tabular}{|c|c|c|c|c|c|c|c|c|c|}
\hline Localidad & Fecha & Experimento & Estadio & Réplicas & $\mathrm{n}$ & $\mathrm{TM}\left(\mathrm{d}^{-1}\right)$ & $g\left(\mathrm{~d}^{-1}\right)$ & $\operatorname{TM}\left(\mathrm{d}^{-1}\right)( \pm)$ & $g\left(\mathrm{~d}^{-1}\right)( \pm)$ \\
\hline \multirow[t]{9}{*}{ Bahía de Mejillones } & $08 / 02 / 10$ & TM 1 & $\mathrm{C} 1$ & 1 & 8 & 0,4 & 0,4 & & \\
\hline & $08 / 02 / 10$ & TM 1 & $\mathrm{C} 3$ & 1 & 7 & 0,2 & 0,1 & & \\
\hline & $08 / 02 / 10$ & TM 1 & $\mathrm{C} 4$ & 3 & 39 & 0,4 & 0,2 & $0,29 \pm 0,17$ & $0,15 \pm 0,09$ \\
\hline & $10 / 02 / 10$ & TM 2 & $\mathrm{C} 2$ & 2 & 7 & 0,4 & 0,3 & $0,28 \pm 0,21$ & $0,20 \pm 0,15$ \\
\hline & $10 / 02 / 10$ & TM 2 & $\mathrm{C} 3$ & 6 & 58 & 0,2 & 0,1 & $0,43 \pm 0,25$ & $0,23 \pm 0,13$ \\
\hline & $21 / 04 / 10$ & TM 3 & $\mathrm{C} 5$ & 4 & 55 & 0,4 & 0,2 & $0,40 \pm 0,05$ & $0,23 \pm 0,03$ \\
\hline & $19 / 07 / 11$ & TM 4 & $\mathrm{C} 5$ & 2 & 29 & 0,4 & 0,2 & $0,40 \pm 0,01$ & $0,23 \pm 0,01$ \\
\hline & $05 / 08 / 11$ & TM 5 & $\mathrm{C} 3$ & 2 & 20 & 0,4 & 0,2 & $0,58 \pm 0,17$ & $0,29 \pm 0,09$ \\
\hline & $05 / 08 / 11$ & TM 5 & $\mathrm{C} 4$ & 2 & 20 & 0,8 & 0,4 & $0,63 \pm 0,18$ & $0,31 \pm 0,09$ \\
\hline \multirow[t]{25}{*}{ Bahía de Concepción } & $11 / 08 / 09$ & TM 1 & $\mathrm{C} 4$ & 1 & 6 & 0,7 & 0,4 & & \\
\hline & $11 / 08 / 09$ & TM 1 & $\mathrm{C} 5$ & 1 & 7 & 0,5 & 0,3 & & \\
\hline & 08/10/09 & TM 2 & $\mathrm{C} 1$ & 1 & 4 & 1,0 & 0,5 & & \\
\hline & 08/10/09 & TM 2 & $\mathrm{C} 2$ & 1 & 6 & 1,1 & 0,8 & & \\
\hline & 08/10/09 & TM 2 & $\mathrm{C} 3$ & 1 & 6 & 0,9 & 0,5 & & \\
\hline & 08/10/09 & TM 2 & $\mathrm{C} 4$ & 1 & 14 & 0,3 & 0,2 & & \\
\hline & $25 / 10 / 09$ & TM 3 & $\mathrm{C} 1$ & 1 & 11 & 0,1 & 0,1 & & \\
\hline & $25 / 10 / 09$ & TM 3 & $\mathrm{C} 2$ & 1 & 4 & 0,3 & 0,2 & & \\
\hline & $25 / 10 / 09$ & TM 3 & $\mathrm{C} 3$ & 2 & 9 & 0,3 & 0,2 & $0,40 \pm 0,16$ & $0,23 \pm 0,07$ \\
\hline & $25 / 10 / 09$ & TM 3 & $\mathrm{C} 4$ & 2 & 12 & 0,3 & 0,2 & $0,17 \pm 0,24$ & $0,08 \pm 0,12$ \\
\hline & $25 / 10 / 09$ & TM 3 & $\mathrm{C} 5$ & 2 & 19 & 0,4 & 0,2 & $0,37 \pm 0,05$ & $0,21 \pm 0,03$ \\
\hline & $22 / 08 / 11$ & TM 4 & $\mathrm{C} 3$ & 1 & 10 & 0,6 & 0,3 & & \\
\hline & $22 / 08 / 11$ & TM 4 & $\mathrm{C} 4$ & 1 & 11 & 0,3 & 0,2 & & \\
\hline & $22 / 08 / 11$ & TM 4 & C5 & 2 & 24 & 0,4 & 0,2 & $0,41 \pm 0,03$ & $0,24 \pm 0,02$ \\
\hline & $22 / 08 / 11$ & TM 4 & $\mathrm{C} 5$ & 1 & 12 & 0,4 & 0,2 & & \\
\hline & $22 / 08 / 11$ & TM 4 & $\mathrm{C} 4$ & 1 & 12 & 0,4 & 0,2 & & \\
\hline & $01 / 09 / 11$ & TM 5 & $\mathrm{C} 3$ & 1 & 11 & 0,8 & 0,4 & & \\
\hline & $21 / 01 / 12$ & TM 6 & $\mathrm{C} 3$ & 1 & 10 & 0,1 & 0,1 & & \\
\hline & $21 / 01 / 12$ & TM 6 & C5 & 3 & 30 & 0,6 & 0,3 & $0,45 \pm 0,10$ & $0,26 \pm 0,06$ \\
\hline & $25 / 01 / 12$ & TM 6 & $\mathrm{C} 4$ & 1 & 10 & 0,7 & 0,3 & & \\
\hline & $25 / 02 / 12$ & TM 6 & $\mathrm{C} 5$ & 2 & 20 & 0,4 & 0,2 & $0,38 \pm 0,01$ & $0,22 \pm 0,01$ \\
\hline & $25 / 02 / 12$ & TM 6 & $\mathrm{C} 4$ & 1 & 10 & 0,9 & 0,4 & & \\
\hline & $26 / 01 / 12$ & TM 7 & $\mathrm{C} 1$ & 1 & 10 & 0,4 & 0,4 & & \\
\hline & $28 / 01 / 12$ & TM 8 & $\mathrm{C} 1$ & 2 & 30 & 0,4 & 0,4 & $0,41 \pm 0,03$ & $0,37 \pm 0,03$ \\
\hline & $28 / 01 / 12$ & TM 8 & $\mathrm{C} 2$ & 3 & 30 & 0,4 & 0,3 & $0,42 \pm 0,04$ & $0,30 \pm 0,03$ \\
\hline
\end{tabular}

En el norte de Chile, el estadio C1 presentó el mayor porcentaje de organismos vivos en la capa de mezcla (100\%) y ZMO (95\%), mientras que el $100 \%$ de los organismos encontrados en la oxiclina fueron organismos muertos (Fig. 4a, b y c). En el estadio C2, se encontró organismos vivos en los 3 estratos, siendo más abundante en la capa de mezcla (100\%) y ZMO (96\%), con un $43 \%$ de los organismos muertos en la oxiclina (Fig. 4a, b y c). El estadio C3, se encontró organismos vivos en los tres estratos ( $>90 \%$ ) (Fig. 4c). El estadio C4, las mayores proporciones de organismos vivos se encontraron en la capa de mezcla (99\%) y oxiclina (95\%), mientras que los organismos muertos aumentaron en la ZMO (24\%) (Fig. 4a, b y c). En el estadio C5, los organismos vivos se encontraron en mayor abundancia en la capa de mezcla (Fig. 4a), mientras que la proporción de organismos muertos aumentó en la oxiclina y ZMO (Fig. 4b y c). Finalmente, la distribución de los individuos muertos y vivos en los adultos, se presentó con un marcado aumentó de organismos muertos en la ZMO, alcanzando proporciones superiores a $25 \%$ de los organismos muertos (Fig. 4a, b y c).

En la zona centro/sur en Concepción, la distribución general de los organismos vivos y muertos mostró un 
patrón similar en todos los estadios, con aumento de la abundancia de individuos muertos bajo la capa de mezcla (Fig. 4). En el estadio C1, fue el estadio que mostró las mayor abundancia de organismos muertos, solamente se presentó un bajo porcentaje de organismos vivos en la capa de mezcla (Fig. 4d) y el 100\% de los organismos encontrados en la oxiclina y ZMO fueron organismos muertos (Fig. 4e y f). En el estadio C2, se encontraron organismos vivos en los 3 estratos, siendo más abundante en la capa de mezcla y con un $40 \%$ de los organismos muertos en la oxiclina y ZMO (Fig. 4d, e y f). En el estadio $\mathrm{C} 3$, se encontraron las mayores abundancias de los organismos vivos en las dos capas superiores $(<90 \%)$, mientras que en la ZMO los organismos vivos disminuyeron a un 60\% aproximadamente (Fig. 4d, e y f). El estadio C4 presentó un patrón similar al estadio C3 (Fig. 4d, e y f). En el estadio C5 la proporción de organismos muertos se encontraron en los 3 estratos con abundancias relativamente similares (Fig. 4d, e y f). Finalmente, la distribución de los estadios adultos presentaron un marcado aumento en la ZMO, donde se registraron valores sobre el $60 \%$ de los organismos muertos (Fig. 4d, e y f).

El test de Kruskal-Wallis mostró diferencias en la abundancia relativa de los organismos vivos asociadas a variaciones del contenido de oxígeno disuelto (OD) $(\mathrm{H}=$ 8,89; $P<0,05)$ y la temperatura $(\mathrm{H}=9,09 ; P<0,05)$ en ambas zonas de estudio.
ASOCIACIÓN DE LA VARIABILIDAD OCEANOGRÁFICA Y LA PROPORCIÓN DE ORGANISMOS MUERTOS DE PARACALANUS INDICUS

Para asociar la distribución de la proporción de los organismos muertos de P. indicus, y las condiciones oceanográficas de los estratos para la zona norte y centro/ sur de Chile definidos previamente en la Tabla 1, se estimaron los valores promedios de temperatura y concentración de oxígeno disuelto para cada uno de los estratos de las estaciones muestreadas en la zona norte (Mejillones) y en la zona centro/sur (Concepción). La Figura 5 representa gráficamente la distribución de los organismos muertos en cada estrato para la zona norte y centro/sur de Chile. La proporción de abundancia de los organismos muertos respecto al total de la abundancia de la población muestreada de $P$. indicus, se incrementó hacia las capas más profundas (oxiclina y ZMO), tanto para la asociación con la temperatura (Fig. 5a) y para el contenido de oxígeno disuelto (Fig. 5b). El análisis de Kruskal-Wallis mostró que existe un efecto de la estratificación de la columna de agua en ambas zonas sobre la proporción de los organismos muertos $(P<0,05)$. Además, se detectaron diferencias significativas en la razón de los organismos muertos y el total de su población muestreada de $P$. indicus con el contenido de oxígeno disuelto y con la temperatura $(H=9,73 ; P<0,05)$ en la zona de norte (Mejillones), aunque esta variación no fue significativa $(P>0,05)$ en la zona centro/sur.
Figura 5. Asociación entre la variabilidad oceanográfica con: a) proporción de organismos muertos de Paracalanus indicus respecto al total en relación a la temperatura (oC) y b) proporción de los copépodos muertos con respecto al total en relación al oxígeno disuelto $\left(\mathrm{ml} \mathrm{L}^{-1}\right)$, en el norte $\left(\sim 23^{\circ} \mathrm{S}\right)$ y centro/sur de Chile ( $\sim 36^{\circ}$ S) / Association between oceanographic variability with: a) the dead copepods percent of Paracalanus indicus on total abundance associated to temperature $(\stackrel{\circ}{C})$ and $b$ ) dead copepods percent on total abundance associated to dissolved oxygen $\left(\mathrm{ml} \mathrm{L}^{-1}\right)$ in zone off northern $\left(\sim 23^{\circ} \mathrm{S}\right)$ and central/southern Chile $\left(\sim 36^{\circ} \mathrm{S}\right)$
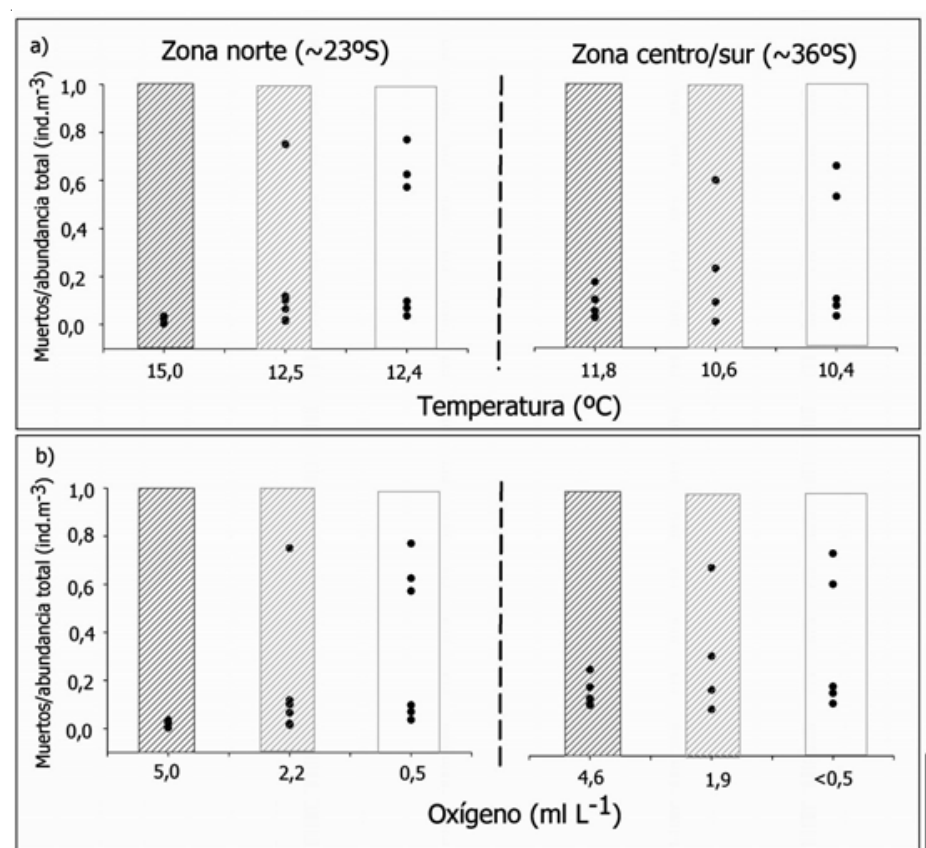

Capa de Mezcla

Oxidina ZMO 


\section{Tasa de Desarrollo de Paracalanus indicus}

La Tabla 4 resume los datos obtenidos en experimentos realizados para obtener tasas de muda, de desarrollo y de crecimiento $(g)$, de $P$. indicus para ambas zonas de estudio. En el norte de Chile $\left(\sim 23^{\circ} \mathrm{S}\right)$ las tasas de crecimiento $(\mathrm{g})$ de $P$. indicus estuvieron entre 0,12 a $0,36 \mathrm{~d}^{-1}$, con un promedio estimado considerando todos los estadios de $0,23 \mathrm{~d}^{-1}$, siendo la tasa del estadio C1, la que presentó el mayor valor. En el centro/sur de Chile $\left(\sim 36^{\circ} \mathrm{S}\right)$, las tasas de crecimiento C- específicas $(g)$ de $P$. indicus estuvieron entre $0,17-0,34 \mathrm{~d}^{-1}$, siendo el estadio C2, el que presentó el mayor valor. Las tasas estimadas para cada estadio de copepodito fueron en promedio 0,25 d $\mathrm{d}^{-1}$. El ANOVA mostró que existen diferencias de las tasas de crecimiento entre ambas zonas de estudio ( $\mathrm{F}=2,70 ; P=0,040$ ) (Tabla 5), siendo el estadio C1 el que representó la mayor diferencia entre las tasas de los estadios, principalmente entre los estadios C4 y C5 (test a posteriori de Tukey, $P<0,05$ ).

\section{Estimación de BIOMASA Y PRODUCCIÓN DE PARACALANUS INDICUS MEDIANTE TASAS DE DESARROLLO}

Para evidenciar cambios de la abundancia y producción de $P$. indicus, se estimó su biomasa, como indicador de estos cambios. La biomasa total estimada en el norte de Chile (Mejillones, $23^{\circ} \mathrm{S}$ ) fue de $160 \mathrm{mg} \mathrm{C} \mathrm{m}^{-2}$, mientras que en el centro/sur de Chile (Concepción, $\sim 36^{\circ} \mathrm{S}$ ), $P$. indicus presentó una biomasa de la población total de aproximadamente $79 \mathrm{mg} \mathrm{C} \mathrm{m}^{-2}$.

Se estimó la producción (mg $\mathrm{C} \mathrm{m}^{-2} \mathrm{~d}^{-1}$ ) del copépodo $P$. indicus a partir del valor de $g$ y el contenido de carbono para cada uno los estadios de desarrollo, determinados por su abundancia y su biomasa (peso seco) en el norte de Chile (Mejillones, $\sim 23^{\circ} \mathrm{S}$ ) y en el centro/sur de Chile (Concepción, $\sim 36^{\circ} \mathrm{S}$ ) de Chile. Se detectó que las tasas de producción fueron $\sim 35 \mathrm{mg} \mathrm{C} \mathrm{m}^{-2} \mathrm{~d}^{-1}$ en Mejillones, mientras que en Concepción fueron de $20 \mathrm{mg} \mathrm{C} \mathrm{m}^{-2} \mathrm{~d}^{-1}$.

\section{Flujos de CARbono vía mortalidad nAtural de ParacalanUS INDICUS HACIA LA ZMO}

El número de organismos totales que va muriendo por día fueron de 424,2 y 81,6 ind. $\mathrm{m}^{-2} \mathrm{~d}^{-1}$, para la zona norte $\mathrm{y}$ centro/sur de Chile, respectivamente. La biomasa por día fue de 1,22 mg C m $\mathrm{m}^{-1}$ en el norte y 0,24 mg C m $\mathrm{m}^{-2} \mathrm{~d}^{-1}$ en el centro/sur de Chile. Al relacionar este valor con la producción poblacional en el norte de Chile (35 mg C m $\mathrm{d}^{-1}$ ) obtenida mediante las tasas de desarrollo, se estimó que el porcentaje de organismos muertos por día correspondió a un 3,4\%. En la zona centro/sur de Chile, al relacionar el valor de biomasa con la producción poblacional (20 $\mathrm{mg} \mathrm{C} \mathrm{m}^{-2} \mathrm{~d}^{-1}$ ), se estimó que el porcentaje de organismos muertos por día correspondió a un 0,2\%. González et al. (1998), determinaron el total de la sedimentación pasiva de materia orgánica que fluctúa entre 0,125-0,176 g C m $\mathrm{d}^{-2} \mathrm{~d}^{-1}$ en la zona norte de la Corriente de Humboldt. En relación a este valor se determinó que el aporte de materia orgánica de $P$. indicus hacia la ZMO en Mejillones es aproximadamente un $0,67 \%$, mientras que en Concepción es aproximadamente un 0,13\% del flujo de $\mathrm{C}$ pasivo derivado del flujo de copépodos muertos.

\begin{tabular}{|c|c|c|c|c|c|c|c|c|c|c|c|}
\hline Localidad & Estadio & $\mathrm{n}$ & & $M(d$ & & & $\left(\mathrm{d}^{-1}\right)$ & & Biomas & $(\mathrm{mg}$ & $\left(\mathrm{m}^{-2}\right)$ \\
\hline \multirow[t]{6}{*}{ Bahía de Mejillones } & $\mathrm{C} 1$ & 8 & 0,40 & \pm & 0,02 & 0,36 & \pm & 0,02 & 0,001 & \pm & 0,001 \\
\hline & C2 & 15 & 0,18 & \pm & 0,21 & 0,20 & \pm & 0,15 & 0,002 & \pm & 0,005 \\
\hline & C3 & 85 & 0,42 & \pm & 0,24 & 0,22 & \pm & 0,13 & 0,027 & \pm & 0,053 \\
\hline & C4 & 59 & 0,40 & \pm & 0,23 & 0,20 & \pm & 0,12 & 0,189 & \pm & 0,316 \\
\hline & C5 & 84 & 0,40 & \pm & 0,04 & 0,23 & \pm & 0,02 & 0,862 & \pm & 1,484 \\
\hline & $\mathrm{AD}$ & & & & & & & & 8,061 & \pm & 13,41 \\
\hline \multirow[t]{6}{*}{ Bahía de Concepción } & $\mathrm{Cl}$ & 47 & 0,34 & \pm & 0,01 & 0,31 & \pm & 0,01 & 0,001 & \pm & 0,004 \\
\hline & $\mathrm{C} 2$ & 44 & 0,27 & \pm & 0,21 & 0,20 & \pm & 0,15 & 0,015 & \pm & 0,016 \\
\hline & $\mathrm{C} 3$ & 50 & 0,44 & \pm & 0,30 & 0,23 & \pm & 0,16 & 0,196 & \pm & 0,216 \\
\hline & $\mathrm{C} 4$ & 67 & 0,32 & \pm & 0,29 & 0,20 & \pm & 0,14 & 2,425 & \pm & 2,392 \\
\hline & C5 & 106 & 0,37 & \pm & 0,14 & 0,22 & \pm & 0,08 & 2,536 & \pm & 3,616 \\
\hline & $\mathrm{AD}$ & & & & & & & & 0,083 & \pm & 0,204 \\
\hline
\end{tabular}


Tabla 5. ANOVA de una vía para comparar las tasas de crecimiento $(g)$ de Paracalanus indicus, entre estadios de copepoditos, localidades y de cada localidad en zonas de surgencias costeras. ${ }^{* *} \boldsymbol{P}<0,05$ / One-way ANOVA to test the effects of copepodid stages, between and through of upwelling coastal zones on growth rates of Paracalanus indicus. ${ }^{* *} P<0.05$

\begin{tabular}{|c|c|c|c|}
\hline Descriptor & g.l. & F- ratio & $P$ \\
\hline Tasas de crecimiento (estadios) & $\begin{array}{c}4 \\
53\end{array}$ & 2,70 & $0,04 * *$ \\
\hline Tasas de crecimiento (localidad) & $\begin{array}{c}1 \\
56\end{array}$ & 2,27 & 0,137 \\
\hline $\begin{array}{r}\text { Tasas de crecimiento (zona norte, } 23^{\circ} \mathrm{S} \text { ) } \\
\text { error }\end{array}$ & $\begin{array}{c}4 \\
19\end{array}$ & 0,65 & 0,636 \\
\hline $\begin{array}{r}\text { Tasas de crecimiento (zona centro/sur, } 36^{\circ} \mathrm{S} \text { ) } \\
\text { error }\end{array}$ & $\begin{array}{c}4 \\
29\end{array}$ & 1,79 & 0,159 \\
\hline
\end{tabular}

** hay diferencias significativas

\section{Discusión}

Los estudios sobre la cuantificación de mortalidad natural in situ, siguen siendo un desafío mayor para la ecología del zooplancton. Entre las razones comunes están que es imposible evaluar la misma población en el tiempo; la advección siempre predomina por sobre los demás procesos de pérdida y ganancia; las agregaciones generan una baja precisión en las estimaciones de abundancia; la historia de vida es inalcanzable; la edad de los organismos o la duración de los estadios necesarios para las estimaciones de mortalidad, son desconocidos, además de que los modelos existentes hasta ahora ocultan parámetros y supuestos innumerables, otorgando error y sensibilidad excesiva a las estimaciones (Ohman 2012). Ciertamente, la depredación es la principal causa de mortalidad natural en la columna de agua (Ohman 2012), no obstante existen muchas otras causas que inducen mortalidad del zooplancton. Entre ellas, para un sistema de surgencia, se sugiere que la ZMO podría incrementar la mortalidad natural de organismos epipelágicos aeróbicos (Escribano et al. 2009). Es importante considerar que en este estudio una fracción de la mortalidad natural, que es aquella determinada por cualquier factor menos la depredación y que induce el transporte vertical pasivo de organismos muertos. En este estudio, se usó la tinción ajustada de rojo neutro para distinguir los organismos entre muertos y vivos de la especie de copépodo Paracalanus indicus, según su estado activo metabólico (Fleming \& Coughlan 1978), es la primera aproximación de mortalidad natural in situ en poblaciones planctónicas en el $\mathrm{SCH}$. $\mathrm{Al}$ respecto, este estudio no determina el flujo vertical de mudas, sino cuerpos de individuos muertos.
Las mudas no representan mortalidad, sino solo el proceso de crecimiento y desarrollo. Por otra parte, la escala de estimación diaria de flujo vertical utilizada en este estudio puede ser menor que la escala de procesos de degradación de individuos muertos, aunque no se descarta procesos de necrofagia que podrían remover individuos muertos antes de ser decantados. Al respecto, Elliott et al. (2010), sugieren el proceso de decantación puede sin embargo superar la degradación y necrofagia. De todas formas, no se puede descartar necrofagia y en tal caso, su existencia afecta el flujo vertical, aunque sí podría incrementar las estimaciones de mortalidad.

La especie $P$. indicus es un componente clave en la estructura y función de la comunidad de copépodos en las zonas costeras del SCH (Escribano \& Hidalgo 2000, Escribano et al. 2007, Hidalgo et al. 2010), debido a su alta abundancia y dominancia durante todo el año (Hidalgo \& Escribano 2001). Sin embargo, esta especie solo ha sido estudiada recientemente, en términos de aporte en el flujo del carbono y estimaciones de biomasa (Escribano et al. 2009). Las mayores abundancias encontradas de $P$. indicus se presentaron en la capa superficial, por sobre los 20-30 m de profundidad, coincidente con lo esperado para la distribución vertical de esta especie, la cual ha sido encontrada en aguas superficiales sobre los $50 \mathrm{~m}$ de profundidad y restringida su distribución vertical a la capa oxigenada en zonas de mínimo de oxígeno (Hidalgo \& Escribano 2001, Escribano et al. 2009). Sin embargo, en esta capa, presenta una partición en la distribución vertical de sus abundancias entre la capa de mezcla y la oxiclina (Tabla 2; Figs. 3 y 4), coincidente con los estudios previos, 
tales como en Perú (Judkins 1979) en el norte de Chile (Escribano et al. 2009), y en el Golfo de México (Kimmel et al. 2010). Es por ello que la ZMO ha sido señalada como una barrera físico-química para la distribución y migración vertical de varias especies de copépodos, en especial en P. indicus (Escribano et al. 2000, 2009, Hidalgo \& Escribano 2001, Hidalgo et al. 2010, 2012). Estos resultados, son concordantes con los valores registrados de abundancia y dominancia de $P$. indicus, desde los estudios de Hidalgo \& Escribano (2001) y recientemente lo registrado por Hidalgo et al. (2010) y Escribano et al. (2012). Cabe señalar que los tipos de redes empleados en este estudio, y los estudios realizados en los últimos 30 años en el SCH (Escribano \& Hidalgo 2000, Hidalgo \& Escribano 2011, Escribano et al. 2012), corresponden a las metodologías clásicas de estudios de zooplancton, en el cual se muestra el mesozooplancton con redes de $200 \mu \mathrm{m}$ de abertura de malla (Harris et al. 2000). No obstante, en este estudio al igual que en otros previos (Escribano et al. 2007), se utilizó adicionalmente la red de $110 \mu \mathrm{m}$ para capturar estadios más pequeños (C1, C2), que además de ser escasos (altas tasas de mudas), podrían no ser apropiadamente muestreados por la red de $200 \mu \mathrm{m}$. En general, no se han obtenido diferencias significativas en abundancias de estadios de esta especie entre ambas tramas de redes. Para este estudio, por otra parte cualquier efecto del arte de muestreo no debería ser diferencial entre individuos vivos y muertos, de tal manera que las estimaciones de proporciones vivos/muertos se pueden presumir como insesgadas.

En relación a las estimaciones de las tasas de crecimiento $(g)$ tanto en la zona norte como centro/sur de Chile, se encontró que no existen diferencias significativas en las tasas de crecimiento entre ambas zonas, a pesar de las evidentes diferencias en la frecuencia de los eventos de surgencia (intermitente y estacional, respectivamente), esto sugiere que quizás un ajuste fisiológico permite a esta especie mantener altas tasas de crecimiento en ambientes altamente heterogéneos como las zonas de surgencia a lo largo de la costa. Al mismo tiempo, se evalúa las diferencias entre cada estadio de desarrollo, se observó que existen diferencias significativas entre ellos (ANOVA una vía, $P<0,05$ ) y un análisis a posteriori (Tukey, $P<0,05$ ) determinó que estas diferencias significativas están marcada entre los estadios tempranos de desarrollo (C1) y los tardíos (C4 y C5). Así, es probable que la producción poblacional de esta especie esté siendo regulada por estos estadios.
El copépodo Paracalanus indicus, a pesar de ser una especie con altos niveles de abundancias y dominancia durante todo el año en las zonas de surgencia en las costas de Chile (Escribano \& Hidalgo 2000, Hidalgo \& Escribano 2001, Morales et al. 2010, Hidalgo et al. 2010), su estrategia de sobrevivencia, es desconocida.

Los estudios de abundancia poblacional del zooplancton marino raramente consideran el estado vital de los organismos al momento del muestreo. Sin embargo, los individuos muertos pueden comprender un $10 \%$ o más del total de los individuos muestreados (Elliott \& Tang 2009). Las diferencias en las abundancias entre organismos vivos y muertos fueron determinadas sus patrones de distribución vertical revelaron claramente que a medida que disminuye la abundancia de organismos vivos, los individuos muertos aumentan en función de la profundidad, específicamente en las capas definidas como oxiclina y ZMO, en Mejillones y Concepción (Tabla 2 y Figs. 3, 4 y 5). El mismo patrón se encontró al observar la mortalidad relativa en función de la profundidad. Sin embargo, sólo se observaron diferencias significativas (Kruskal Wallis, $\mathrm{H}=9,73 ; P<0,05$ ), en la proporción de organismos muertos por el total en Mejillones.

La abundancia de individuos muertos para cada estadio fue similar para ambas zonas (Fig. 4), las diferencias se observaron entre los estadios, y principalmente fueron marcadas por los estadios tempranos (C1 y C2), donde los valores más altos de mortalidad se encontraron en la oxiclina y ZMO. La mortalidad puede variar con la ontogenia, ya que los metazoos experimentan diferentes riesgos y pérdidas en distintos puntos de su historia de vida, por ejemplo, la masa corporal puede variar hasta 3 órdenes de magnitud desde los estadios tempranos hacia los adultos para muchos tipos de holoplancton, estos cambios tienen que ver con el comportamiento natatorio, las respuestas de escape, hidrodinámica y visibilidad (Ohman 2012). Es probable entonces, que las tasas de mortalidad varíen con la etapa de desarrollo y tamaño del organismo.

En este estudio se observó que la biomasa de organismos muertos fue más alta en Mejillones (1,22 mg $\left.C \mathrm{~m}^{-2} \mathrm{~d}^{-1}\right)$ que en Concepción $\left(0,24 \mathrm{mg} \mathrm{C} \mathrm{m}^{-2} \mathrm{~d}^{-1}\right)$, estos valores en relación a la producción total de la población, indican que en total un 3,4\% y 1,2\% muere diariamente en Mejillones y Concepción, respectivamente. La alta variabilidad de la surgencia costera y la heterogeneidad de estos ambientes, tales como el área de Mejillones, pueden tener mayor impacto en el tamaño poblacional de 
las poblaciones planctónicas, aun cuando estas presenten altas abundancias, como es el caso de $P$. indicus. Sin embargo, esta especie puede compensar su tamaño poblacional con altas tasas de crecimiento, que han mostrado ser diferentes entre sus estadios (C1, C4 y C5). A diferencia de la zona centro/sur, donde las condiciones oceanográficas obedecen a un patrón marcadamente estacional, las tasas de mortalidad pueden ser afectadas por otros factores, tales como cantidad y calidad de alimento, que no obstante no estarían drásticamente afectando sus tasas de mortalidad diaria, mostrando así, proporciones menores de los organismos muertos en relación al total de la población muestreada.

Sin embargo, algunos estudios recientes indican que los copépodos muertos representan una fracción importante $(\sim 1 / 4-1 / 2)$ del total del flujo pasivo de carbono orgánico particulado hacia aguas profundas (Sampei et al. 2009). Elliott et al. (2010) estimó que los individuos muertos de $A$. tonsa aportan un valor $<0,3 \%$ a la sedimentación pasiva del estuario York River (Virginia, USA). González et al. (1998), determinaron el total de la sedimentación pasiva de materia orgánica que fluctúa 0,125-0,176 g C m $\mathrm{d}^{-1}$ en la zona norte de Chile. En relación a este valor, se determinó que en la zona norte de Chile el aporte de materia orgánica de $P$. indicus es de $0,67 \%$ derivado del flujo de copépodos muertos. Mientras que en la zona centro/sur de Chile el aporte de materia orgánica de $P$. indicus hacia la ZMO es de un $0,13 \%$ derivado del flujo de copépodos muertos. Esto indica que a pesar del bajo porcentaje de individuos muertos con respecto al total de la población, el copépodo $P$. indicus puede representar un importante porcentaje del flujo pasivo de C, y por tanto es un importante aporte de materia orgánica hacia la ZMO. Por otra parte, en este estudio solo se consideró una especie de pequeño tamaño, por lo cual si se considerara la comunidad en conjunto, es de esperar un flujo vertical pasivo de C orgánico muy sustantivo por parte del zooplancton.

\section{Agradecimientos}

Este trabajo ha sido financiado por los proyectos FONDECYT 1080037 y 11090146 otorgados a R. Escribano y P. Hidalgo, respectivamente. Agradecemos al equipo del laboratorio PLAMZ en Concepción y especialmente al equipo de trabajo en Antofagasta. A la tripulación de 'Kay Kay II' y a don Juan Menares por su valiosa colaboración durante los muestreos. Al Programa FONDAP- COPAS de la Universidad de Concepción. A los evaluadores anónimos por sus comentarios del presente manuscrito.

\section{LITERATURA CITADA}

Bickel S \& K Tang. 2010. Microbial decomposition of proteins and lipids in copepod versus rotifer carcasses. Marine Biology 157: 1613-1624.

Chisholm L \& J Roff. 1990. Size-weight relationships and biomass of tropical neritic copepods off Kingston, Jamaica. Marine Biology 106: 71-77.

Elliott D \& W Tang. 2009. Simple staining method for differentiating live and dead marine zooplankton in field samples. Limnology and Oceanography: Methods 7: 585594.

Elliott D, T Courtney, K Harris \& W Tang. 2010. Dead in the water: The fate of copepod carcasses in the York River estuary, Virginia. Limnology and Oceanography 55: 18211834.

Escribano R. 1998. Population dynamics of Calanus chilensis in the Chilean eastern boundary Humboldt Current. Fisheries Oceanography 7: 241-251.

Escribano R \& I McLaren. 1999. Production of Calanus chilensis from the upwelling area of Antofagasta, northern Chile. Marine Ecology Progress Series 177: 147-156.

Escribano R \& P Hidalgo. 2000. Spatial distribution of copepods in the north of the Humboldt Current region off Chile during coastal upwelling. Journal of the Marine Biological Association of the United Kingdom 80: 283290.

Escribano R, V Marín \& P Hidalgo. 2001. The influence of coastal upwelling on the distribution of Calanus chilensis in the Mejillones Peninsula (northern Chile): implications for its population dynamics. Hidrobiología 453/454: 143151.

Escribano R, D Daneri, L Farías, V Gallardo, H González, D Gutierrez, C Lange, C Morales, O Pizarro, O Ulloa \& M Braun. 2004. Biological and chemical consequences of the 1997-1998 El Niño in the Chilean coastal upwelling system: a synthesis. Deep-Sea Research II 51: 2389-2411.

Escribano R, P Hidalgo, H González, R Giesecke, R Riquelme- Bugueño \& K Manriquez. 2007. Interannual and seasonal variability of metazooplancton in the Central/ South upwelling region off Chile. Progress in Oceanography 75: 470-485.

Escribano R, P Hidalgo \& C Krautz. 2009. Zooplankton associated with the oxygen minimum zone system in the northern upwelling region of Chile during March 2000. Deep-Sea Research II 56: 1083-1094.

Fleming JM \& J Coughlan. 1978. Preservation of vitally stained zooplankton for live/dead sorting. Estuaries 1(2): 135-137.

Frangoulis C, D Christou \& J Hecq. 2005. Comparison of marine copepod outfluxes: Nature, rate, fate and role in the carbon and nitrogen cycles. Advances in Marine Biology 47: 253-309. 
González H, G Daneri, D Figueroa, J Iriarte, N Lefevre, G Pizarro, R Quiñones, M Sobarzo \& A Troncoso. 1998. Producción primaria y su destino en la trama trófica pelágica y océano profundo e intercambio océano-atmósfera de CO2 en la zona norte de la Corriente de Humboldt $\left(23^{\circ} \mathrm{S}\right)$ : Posibles efectos del evento El Niño, 1997-98 en Chile. Revista Chilena de Historia Natural 71: 429-458.

Hidalgo P \& R Escribano. 2001. Succession of pelagic-copepod species during the period 1996/1998 in northern Chile: the influence of the 1997-98 El Niño. Hydrobiología 453: 153160.

Hidalgo P, R Escribano \& C Morales. 2005. Ontogenetic vertical distribution and diel migration of the copepod Eucalanus inermis in the oxygen minimum zone off northern Chile. Journal of Plankton Research 27: 519-529.

Hidalgo P \& R Escribano. 2007. The coupling of life cycles of the copepods Calanus chilensis and Centropages brachiatus to upwelling variability in the Central-South upwelling region off Chile. Progress in Oceanography 75: 501-517.

Hidalgo P \& R Escribano. 2008. The life cycles of two coexisting copepods, Calanus chilensis and Centropages brachiatus, in the upwelling zone off northern Chile $\left(23^{\circ}\right.$ S). Marine Biology 155: 429-442.

Hidalgo P, R Escribano, O Vergara, E Jorquera, K Donoso \& $\mathbf{P}$ Mendoza. 2010. Patterns of copepod diversity in the Chilean coastal upwelling system. Deep-Sea Research II 57: 2089-2097.

Hidalgo P, R Escribano, M Fuentes, E Jorquera \& O Vergara. 2012. How coastal upwelling inûuences spatial patterns of size-structured diversity of copepods off central-southern Chile (summer 2009). Progress in Oceanography 92-95: 134-145.

Kimmel D, W Boicourt, J Pierson, M Romam \& X Zhang. 2010. The vertical distribution and diel variability of mesozooplankton biomass, abundance and size in response to hypoxia in the northern Gulf of Mexico USA. Journal of Plankton Research 33: 1185-1202.

Lee B \& N Fisher. 1994. Effects of sinking and zooplankton grazing on the release of elements from planktonic debris. Marine Ecology Progress Series 110: 271-281.
Morales C, ML Torreblanca, S Hormazabal, M CorreaRamírez, S Nuñez \& P Hidalgo. 2010. Mesoscale structure of copepod assemblages in the coastal transition zone and oceanic waters off central-southern Chile. Progress in Oceanography 84: 158-173.

Ohman M. 2012. Estimation of mortality for stage-structured zooplankton populations: What is to be done? Journal of Marine Systems 93: 4-10.

Parsons TR, Y Maita \& CM Lalli. 1984. A manual of chemical and biological methods for seawater analysis, $173 \mathrm{pp}$. Pergamon Press, Oxford.

Poulet S, M Laabir, A Ianora \& A Miralto. 1995. Reproductive response of Calanus helgolandicus. I. Abnormal embryonic and naupliar development. Marine Ecology Progress Series 129: 85-95.

Poulet S, R Escribano, P Hidalgo, A Cueff, T Wichard, V Aguilera, C Vargas \& G Pohnert. 2007. Collapse of Calanus chilensis reproduction in a marine environment with high diatom concentration. Journal of Experimental Biology and Ecology 352: 187-199.

Runge J \& J Roff. 2000. The measurement of growth and reproductive rates. In: Harris RP, P Wiebe, J Lenz, HR Skjoldal \& M Huntley (eds). ICES zooplankton methodology manual, pp. 401-454. Academic Press, New York.

Sampei M, H Sasaki, H Hattori, A Forest \& L Fortier. 2009. Significant contribution of passively sinking copepods to the downward export flux in Arctic waters. Limnology and Oceanography 54: 1894-1900.

Sobarzo M, R Shearman \& S Lentz. 2007. Near-inertial motions over the continental shelf off Concepción, central Chile. Progress in Oceanography 75: 348-362.

Tang K, C Freund \& C Schweitzer. 2006. Occurrence of copepod carcasses in the lower Chesapeake Bay and their decomposition by ambient microbes. Estuarine, Coastal and Shelf Science 68: 499-508.

Zajaczkowski M \& J Legezynska. 2001. Estimation of zooplankton mortality caused by an Arctic glacier outflow. Oceanologia 43: 341-351.

Recibido el 7 de marzo de 2012 y aceptado el 28 de junio de 2012

Editor Asociado: Mauricio Landaeta D. 